\title{
Toward an Integrative Study of Narcissism
}

\author{
Mark A. Blais and Jessica A. Little \\ Massachusetts General Hospital, Boston, MA, and Harvard Medical School
}

The expression of narcissism spans the continuum from normal to pathological and has meaningful correlates in clinical and nonclinical populations. There is growing speculation that narcissism also contributes to major societal concerns (e.g., terrorism and corporate malfeasance). Improving our understanding of the psychological, interpersonal, and social expressions of narcissism should be one of the most important areas in behavioral science research. Unfortunately, the study of narcissism is fragmented and underpursued (see Miller and Campbell, pp. 180-191, this issue). Pathological narcissism (PN), primarily narcissistic personality disorder (NPD; American Psychiatric Association, 1994), has been studied mainly through clinical case reports and psychodynamic theory (Ronningstam, 2005), whereas the study of trait narcissism has utilized more empirical methods. Miller and Campbell (this issue) contend that the current understanding of $\mathrm{PN}$ is speculative and empirically lacking. His proposed remedy is for psychiatric and clinical researchers to incorporate the strategies and tools used to study trait narcissism. Although research on PN should be more empirically based, the uncritical adoption of the trait narcissism paradigm seems ill advised. Rather, an integrative research perspective incorporating knowledge and methodologies across disciplines would seem more promising.

To see further discussion of the Target Conceptual Article, Commentaries, and Author Response, as well as to contribute to the ongoing dialogue on this topic, please visit our Online Forum at http://pdtrtonline.apa.org.

Mark A Blais and Jessica A. Little, Department of Psychiatry, Massachusetts General Hospital, Boston, MA, and Harvard Medical School.

Correspondence concerning this article should be addressed to Mark A. Blais, Director, Psychological Evaluation and Research Laboratory, Massachusetts General Hospital, One Bowdoin Square, 7th Floor, Boston, MA 02114. E-mail: mblais@partners.org

\section{Trait Narcissism}

The trait narcissism literature is prolific but based almost exclusively on the Narcissistic Personality Inventory (NPI; Raskin \& Hall, 1981). Miller and Campbell (this issue) acknowledge that the NPI has psychometric problems and does not specifically endorse its use in clinical research. However, the NPI and trait narcissism are so interconnected that they have become practically synonymous (Cain, Pincus, $\&$ Ansell, 2008). Given their considerable overlap, it is difficult to judge the relevance of the trait narcissism literature for clinical research without also considering the limitations of the NPI.

The NPI was developed to measure narcissism in nonclinical samples. Despite years of application, the NPI factor structure remains unclear (Kubarych, Deary, \& Austin, 2004), and NPI subscales typically lack adequate psychometric properties (Brown, Budzek, \& Tamborski, 2009). NPI subscales also measure both adaptive and maladaptive qualities (Pincus et al., 2009)—a finding that raises significant questions about the value and meaning of the NPI total score (Brown et al., 2009). Finally, at present, little is known about the utility of the NPI in clinical populations (Pincus et al., 2009). Therefore, although we wholeheartedly encourage increased measurement rigor in studies of PN, the NPI does not seem to be the ideal instrument. However, there are encouraging developments in the area of selfreported narcissism.

Brown et al. (2009) have shown that grandiosity (as measured on the Narcissistic Grandiosity Scale; Rosenthal, Hooley, \& Steshenko, 2007) and entitlement (as measured on the Psychological Entitlement Scale; Campbell, Bonacci, Shelton, Exline, \& Bushman, 2004) form two well-defined dimensions of overt PN. In a series of studies, they found that entitlement and grandiosity functioned independently of one another and evidenced distinct associations with mental health and problematic behaviors. These 
dimensions may begin to differentiate the intrapsychic (grandiosity) and interpersonal (entitlement) features of PN. Furthermore, this measurement approach could appeal to clinical researchers, as features of entitlement and grandiosity are well represented within the NPD criteria set. Exploring associations among the NPD criteria and these brief measures might begin to clarify the constructs underlying both PN and NPD.

Alternatively, the Pathological Narcissism Inventory (PNI; Pincus et al., 2009) is a recently developed self-report measure of PN that measures grandiose and vulnerable affect and self states. In particular, the PNI captures narcissistic vulnerabilities, such as fragile self-esteem and self-devaluation, that are often seen in treatment-seeking patients. Initial PNI results appear promising. We encourage clinical researchers to integrate these and other emerging self-report instruments into their research projects. In addition to improving the empirical foundation of $\mathrm{PN}$, the consistent use of conceptually clear and psychometrically precise measures will allow for the disaggregation of PN into more fundamental psychological constructs. Successful disaggregation of PN will ultimately result in improved diagnostic utility and facilitate theory validation and refinement (for a discussion of the interrelationship of measurement and theory refinement, see Smith, McCarthy, \& Zapolski, 2009).

\section{Pathological Narcissism and Narcissistic Personality Disorder}

There is a large literature demonstrating the psychometric limitations of the personality disorders as given in the Diagnostic and Statistical Manual of Mental Disorders (DSM), including NPD (Blais \& Norman, 1997). Nonetheless, it is important to recognize that DSM criterion sets are not psychological tests and are not intended to measure latent constructs. Rather, the DSM categories are based on the syndrome model, wherein diagnostic criteria represent groups of heterogeneous signs and symptoms that together best identify and differentiate a specific syndrome. Therefore, narrowly focused empirical studies of the NPD criteria are of limited value. For example, factor analytic studies have consistently shown that signs of overt narcissism (grandiosity) are overly represented among the NPD criteria (Blais, Hilsenroth, \& Castlebury, 1997; Miller, Hoffman, Campbell, \& Pilkonis, 2008). However, it is important to note that the DSM system requires that patients be rated for all personality disorder criteria. Thus, although grandiosity may be a feature of all narcissistic patients, subtypes of narcissism (covert or fragile narcissists) might be identified through specific patterns of comorbidity with avoidant, dependent, or borderline criteria. Broadening empirical studies to include the full $D S M$ personality disorder criteria pool might be more informative.

More to the point, clinical research would be greatly enhanced by exploring the real-life functioning and non-DSM characteristics of patients with NPD. Research with the Shedler-Westen Assessment Procedure (SWAP; Shedler \& Westen, 2007), a clinician-rated tool, demonstrates the richness of this approach. A recent SWAP study provided a detailed description of patients with NPD and revealed the construct to be more complex than previous suspected. In fact, three NPD subtypes (grandiose, fragile, and high functioning) were identified (Russ, Shedler, Bradley, \& Westen, 2008). Consistent with the trait narcissism literature, the SWAP subtypes range from high functioning (normal) to pathological. Furthermore, the SWAP findings revealed a form of PN, "the fragile narcissist," that is well represented in the clinical and theoretical literature but underidentified in empirical studies. Other studies using clinician ratings have demonstrated meaningful relationships between normal personality traits (Blais, 1997) and psychodynamic defenses in patients with NPD (Blais, Conboy, Wilcox, \& Norman, 1996). Clearly, including measurement methods beyond self-report scales will be important for understanding the full range and impact of narcissism.

There is a pressing need to advance our knowledge of narcissism; however, it seems unlikely that a single research method or tool is adequately suited to this challenge. Multidisciplinary, integrative research is needed to better understand this complex condition. In closing, we offer the following modest suggestions as a start: (1) Focus on developing conceptually clear and psychometrically precise self-report tools that measure normal and pathological expressions of narcissism. (2) Broaden clinical research to identify real-life functioning and 
non-DSM features of patients with NPD while avoiding narrowly focused studies of the diagnostic criteria. (3) Routinely incorporate multimethod assessments into research on normal and pathological narcissism (Hilsenroth, Handler, \& Blais, 1996). If adopted, these suggestions may begin to yield a more sophisticated and meaningful understanding of narcissism.

\section{References}

American Psychiatric Association. (1994). Diagnostic and statistical manual of mental disorders (4th ed.). Washington DC: Author.

Blais, M. A. (1997). Clinician ratings of the fivefactor model of personality and the $D S M-I V$ personality disorders. Journal of Nervous and Mental Disease, 185, 388-393.

Blais, M. A., Conboy, C. A., Wilcox, N., \& Norman, D. K. (1996). An empirical study of the DSM-IV Defensive Functioning Scale in personality disordered patients. Comprehensive Psychiatry, 3, 435440.

Blais, M. A., Hilsenroth, M. J., \& Castlebury, F. D. (1997). Content validity of the $D S M-I V$ borderline and narcissistic personality disorder criteria sets. Comprehensive Psychiatry, 38, 31-37.

Blais, M. A., \& Norman, D. K. (1997). A psychometric evaluation of the $D S M-I V$ personality disorder criteria. Journal of Personality Disorders, 11, 168-176.

Brown, R. P., Budzek, K., \& Tamborski, M. (2009). On the meaning and measure of narcissism. Personality and Social Psychology Bulletin, 35, 951964.

Cain, N. M., Pincus, A. L., \& Ansell, E. B. (2008). Narcissism at the crossroads: Phenotypic description of pathological narcissism across clinical theory, social/personality psychology, and psychiatric diagnosis. Clinical Psychology Review, 28, 638656.

Campbell, W. K., Bonacci, A. M., Shelton, J., Exline, J. J., \& Bushman, B. J. (2004). Psychological entitlement: Interpersonal consequences and validation of a new self-report measure. Journal of Personality Assessment, 83, 29-45.
Hilsenroth, M. J., Handler, L., \& Blais, M. A. (1996). Assessment of narcissistic personality disorder: A multi-method review. Clinical Psychology Review, 16, 655-683.

Kubarych, T. S., Deary, I. J., \& Austin, E. J. (2004). The Narcissistic Personality Inventory: Factor structure in a non-clinical sample. Personality and Individual Differences, 36, 857-872.

Miller, J. D., \& Campbell, W. K. (2010). The case for using research on trait narcissism as a building block for understanding narcissistic personality disorder. Personality Disorders: Theory, Research, and Treatment, 1, 180-191.

Miller, J. D., Hoffman, B. J., Campbell, W. K., \& Pilkonis, P. A. (2008). An examination of the factor structure of Diagnostic and Statistical Manual of Mental Disorders, fourth edition, narcissistic personality disorder criteria: One or two factors? Comprehensive Psychiatry, 49, 141-145.

Pincus, A. L., Ansell, E. B., Pimentel, C. A., Cain, N. M., Wright, A. G. C., \& Levy, K. N. (2009). Initial construction and validation of the Pathological Narcissism Inventory. Psychological Assessment, 21, 365-379.

Raskin, R., \& Hall, C. S. (1981). The Narcissistic Personality Inventory: Alternate form reliability and further evidence of construct validity. Journal of Personality Assessment, 45, 159-162.

Ronningstam, E. F. (2005). Identifying and understanding the narcissistic personality. New York: Oxford University Press.

Rosenthal, S. A., Hooley, J. M., \& Steshenko, Y. (2007). Distinguishing grandiosity from selfesteem: Development of the Narcissistic Grandiosity Scale. Manuscript in preparation.

Russ, E., Shedler, J., Bradley, R., \& Westen, D. (2008). Refining the construct of narcissistic personality disorder: Diagnostic criteria and subtypes. The American Journal of Psychiatry, 165, 14731481.

Shedler, J., \& Westen, D. (2007). The ShedlerWesten Assessment Procedure (SWAP): Making personality diagnosis clinically meaningful. Journal of Personality Assessment, 89, 41-55.

Smith, G. T., McCarthy, D. M., \& Zapolski, T. C. B. (2009). On the value of homogeneous constructs for construct validation, theory testing, and the description of psychopathology. Psychological Assessment, 21, 272-284. 